PAPER

\title{
Reference data on Japanese vowel devoicing: Effects of speakers' and parents' places of origin and within-speaker reproducibility
}

\author{
Kanae Amino*, Hisanori Makinae, Toshiaki Kamada and Takashi Osanai \\ National Research Institute of Police Science, \\ 6-3-1 Kashiwanoha, Kashiwa, 277-0882 Japan
}

(Received 24 November 2016, Accepted for publication 19 November 2017)

\begin{abstract}
This study provides reference data on vowel devoicing in Japanese spontaneous speech for forensic and other speech investigations. We analysed the running speech of 226 speakers in order to examine how the places of origin of the speakers and their parents influence the occurrence frequency of vowel devoicing. According to the dialect distribution map of vowel devoicing, we classified the speakers into two dialects: the dialect where vowel devoicing occurs frequently $\left(\mathrm{D}_{\mathrm{F}}\right)$ and that where devoicing occurs infrequently $\left(\mathrm{D}_{\mathrm{IF}}\right)$. The results showed that $\mathrm{D}_{\mathrm{F}}$ speakers with $\mathrm{D}_{\mathrm{F}}$ parents devoiced the vowels most frequently, while $\mathrm{D}_{\mathrm{IF}}$ speakers with $\mathrm{D}_{\mathrm{IF}}$ parents devoiced them the least. The devoicing of speakers with parents of different dialects was in between. Some speakers, irrespective of whether they were $\mathrm{D}_{\mathrm{F}}$ or $\mathrm{D}_{\mathrm{IF}}$, showed a percentage of vowel devoicing that contradicted their dialects while keeping the accentuation and intonation of their dialects. We further examined withinspeaker variability in vowel devoicing. We found that the speakers who devoice vowels frequently showed consistent devoicing, whereas those who devoice them infrequently showed occasional and inconsistent devoicing. Forensically, we should not simply judge a speaker's dialect by using the occurrence frequency of vowel devoicing; instead, we should also look at its reproducibility and other dialect-dependent features.
\end{abstract}

Keywords: Devoicing, High vowels, Regional dialect, Dialect estimation, Forensic reference data

PACS number: 43.70.Fq, 43.72.Uv [doi:10.1250/ast.39.207]

\section{INTRODUCTION}

Vowel devoicing is a phenomenon in which vowels are produced without accompanying vocal fold vibration. The occurrence frequency of vowel devoicing (hereafter, FVD) in Japanese depends on various factors, including linguistic and social factors. Among those factors, dialectal variations of FVD have long been investigated [e.g. 1-3]. Also, the details of the regional distribution of vowel devoicing are provided as a dialect map, which is shown in Fig. 1 [4].

Due to the abundance of available previous research and its simplicity of analysis, FVD is one of the prospective speech characteristics used in linguistic profiling, i.e. the dialectal variations seen in FVD can be helpful for forensic linguists to estimate speakers' places of origin. In linguistic profiling, forensic linguists analyse recorded, and sometimes written, language materials; extract dialectal characteristics; and estimate the origin of the speaker, or the writer [5], by using the knowledge of their regional distribution. However, for dialectal characteristics to be

\footnotetext{
*e-mail: amino@nrips.go.jp
}

used in a forensic context, both their regional distribution and their between- and within-speaker variations must be investigated. Forensic speech scientists suggest that when we build forensic reference data, a population of enough size should be included to guarantee data accuracy and the reproducibility of the data must be evaluated [6,7]. Further, we need to have enough data based on the analysis of spontaneous speech, as most acoustic parameters, especially suprasegmentals, may vary between read and spontaneous speech $[6,8]$.

This study provides reference data on FVD of spontaneous Japanese speech for forensics and other speechoriented research. Survey on the related work on Japanese vowel devoicing is summarised in the next section. The procedure and results of the acoustic analyses are described in Sect. 3, where we focused on the relationship between the speakers' places of origin and FVD in their utterances. We also examine the effect of their parents' dialects on their speech. In Sect. 4, we investigate the within-speaker variation of vowel devoicing in order to assess the reproducibility of FVD. Finally, Sect. 5 gives a summary of this study and its conclusions. 


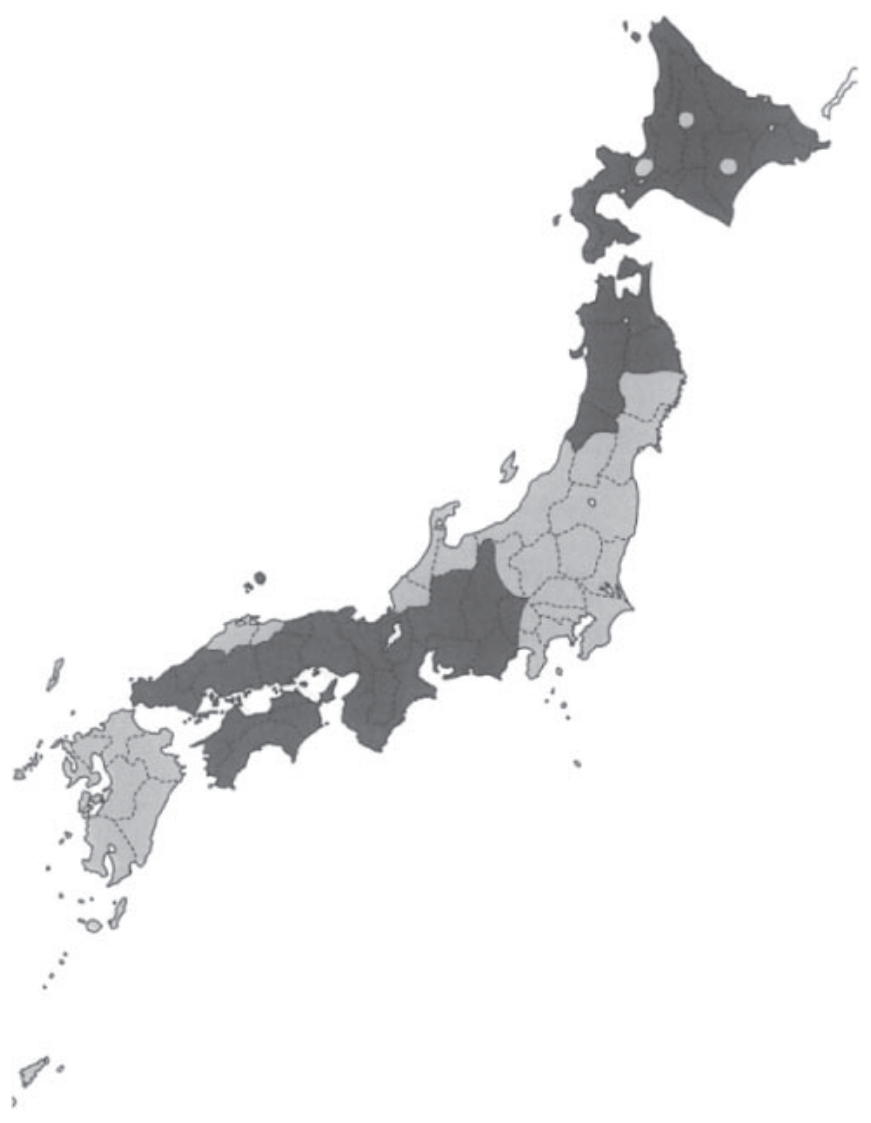

Fig. 1 Regional distribution of dialects in Japan, showing areas where vowel devoicing occurs frequently (light grey) and infrequently (dark grey). Reprinted from [4].

\section{RELATED WORK ON JAPANESE VOWEL DEVOICING}

As mentioned above, FVD in Japanese varies depending on the linguistic and social factors. For example, it is reported that FVD is influenced by the segmental contexts, accent patterns, and positions in a word. Short high vowels that are positioned either between two voiceless consonants or between a voiceless consonant and a pause are most likely to be devoiced [9]. It is known that FVD varies significantly according to the preceding $\left(\mathrm{C}_{1}\right)$ and following $\left(\mathrm{C}_{2}\right)$ consonants. Kawai et al. [10] analysed read words and found that FVD is high when $C_{2}$ is a voiceless plosive or a voiceless affricate. Yoshida [11] analysed words in carrier sentences and found that FVD is lower when both $\mathrm{C}_{1}$ and $\mathrm{C}_{2}$ are voiceless fricatives. Fujimoto [12] confirmed a similar tendency and found FVD to be low especially when $\mathrm{C}_{2}$ was $/ \mathrm{h} /$. Further, some, but not all, studies report that there is a tendency for FVD to be higher when the vowel in the following mora is an open vowel [1,11]. Kawai et al. [10] analysed the inventories of an accent dictionary and proposed vowel devoicing rules for speech synthesis. Firstly they summarised the interactive effects of the segmental contexts, accentuation, and the positions in a word. Then they generalised the linguistic environments that induce vowel devoicing. Their rules can also cope with successive vowel devoicing environments. Many studies including Kawai's [10] pointed out that FVD is lower in accented compared to unaccented mora and higher in word-initial compared to word-medial position [reviewed in 13].

Social properties of speakers also affect FVD. Byun $[1,14]$ analysed words uttered by speakers of different generations and different dialects and found that in most dialects, younger speakers tend to devoice their vowels more frequently than older speakers do. Imai [15] investigated the effects of age, gender, and speech style on FVD and concluded that gender and generation had an interaction; younger male speakers showed the highest FVD, younger female speakers showed the least. Imaizumi et al. [16] suggested that careful speech had less FVD; on the other hand, several studies showed that FVD was quite high in spontaneous speech [e.g. 15,17,18].

Regional dialects are another principal factor influencing FVD. Generally, whereas vowel devoicing occurs by rule in Eastern dialects, it is less common in Western dialects [9,19]. Fujimoto and her colleague [2,12,20] conducted a series of studies on vowel devoicing in Tokyo and Osaka Japanese, which represent Eastern and Western dialects, respectively. They found that the speakers of Osaka Japanese showed more between-speaker variations in FVD and their FVD depended on the speech rate $[2,12]$. Fujimoto [20] explained the dialectal differences in FVD by showing the differences in glottal opening patterns. Fujimoto and Kiritani [2] also reported that some of the Osaka speakers showed an FVD that was similar to Tokyo speakers when the accentuation of the target words was controlled. Okamoto [21] investigated isolated words uttered by eight speakers of Osaka Japanese. He found that FVD varied among the speakers and the factors affecting FVD were different for each speaker. Parents' places of origin are important in dialectology; nonetheless, few studies investigated their effect on FVD. Sugito [3] analysed the FVDs of words uttered by Tokyo and Osaka speakers. She found that FVD may be affected by places of origin of parents' and history of moving to regions of different dialects; however, her study included only one or two speakers for each dialect and it is clearly not enough for showing the existence of the effect.

As mentioned in the previous section, regional distribution of vowel devoicing can be confirmed in the dialect map shown in Fig. 1. Based on FVD, this map divides Japanese dialects into two categories: the dialect where vowel devoicing occurs frequently (hereafter, $\mathrm{D}_{\mathrm{F}}$ ) and the one where it occurs infrequently (hereafter, $\mathrm{D}_{\mathrm{IF}}$ ). Byun [1] suggested that one of the defects of this map was that no clear criterion was given regarding the distinction between 
$\mathrm{D}_{\mathrm{F}}$ and $\mathrm{D}_{\mathrm{IF}}$. Subsequently, she investigated the FVD of isolated words uttered by 608 speakers from 41 prefectures. Her conclusion was that the percentage of vowel devoicing (hereafter, $P_{\mathrm{VD}}$ ) for the distinction between the two categories was approximately $60 \%$. Kawatsu and Maekawa [22] analysed the 'core speech' of the Corpus of Spontaneous Japanese (CSJ) recorded from speakers of $\mathrm{D}_{\mathrm{F}}$ [23]. From their data, $P_{\mathrm{VD}}$ for $\mathrm{D}_{\mathrm{F}}$ speakers was $88.1 \%$ on average. Here, as well, we notice that the speech style, read or spontaneous, influences FVD greatly.

As we have just seen, most of the previous studies on FVD dealt with the analysis of words in isolation or words read in carrier sentences. Moreover, most of them focused on just a few dialects. In order to understand the dialectal variations of vowel devoicing, we analysed spontaneous speech recorded from speakers from various dialectal districts.

\section{DIALECTAL VARIATION OF VOWEL DEVOICING}

\subsection{Analysis Methods}

In this study, Academic Presentation Speech and Simulated Public Speaking of CSJ [23] were used for the analyses. This corpus has a list of speaker information, including place of origin, history of moving, and parents' places of origin. Selection of the target speakers was conducted based on the following criteria: speakers who (1) have lived in only one prefecture at least before they were 12 years old, (2) have no experience of living abroad, and (3) come from the prefectures where $\mathrm{D}_{\mathrm{F}}$ and $\mathrm{D}_{\mathrm{IF}}$ regions are not mixed in Fig. 1 and so do their parents. A total of 226 speakers (163 males and 63 females) were randomly selected from those who meet the criteria and their speech data were analysed. We analysed the first presentation speech for those who contributed speech data several times.

The speakers were first classified into two devoicing categories, $\mathrm{D}_{\mathrm{F}}$ and $\mathrm{D}_{\mathrm{IF}}$, according to their own places of origin and then into three groups in each category according to their parents' places of origin, as shown in Table 1. Table 2 provides a list of the speakers' places of origin. We had $134 \mathrm{D}_{\mathrm{F}}$ speakers and $92 \mathrm{D}_{\mathrm{IF}}$ speakers.

Although CSJ provides detailed annotation including vowel devoicing for 'core-speech,' we analysed all data including 'core-speech' by ourselves in order to make the analysis consistent. We focused on the segmental contexts where FVD is high with the speakers of $D_{F}$. The analysis target was the short high vowel positioned between two voiceless obstruents, $C_{1}$ and $C_{2}$. We excluded the segmental contexts where FVD is less likely to occur, i.e. (1) when $\mathrm{C}_{2}$ is $/ \mathrm{h} /$, (2) when both $\mathrm{C}_{1}$ and $\mathrm{C}_{2}$ are fricatives, (3) when $\mathrm{C}_{1}$ is an affricate and $\mathrm{C}_{2}$ is a fricative, and (4) when the same mora is repeated [11-13]. We did not place any control on the vowel in the second mora.
Table 1 Classification of speakers into groups. $D_{F}$ and $\mathrm{D}_{\mathrm{IF}}$ stand for the dialects where vowel devoicing occurs frequently and infrequently, respectively.

\begin{tabular}{|c|c|c|c|}
\hline \multirow{2}{*}{ Group } & \multicolumn{2}{|c|}{ Dialect } & \multirow{2}{*}{ Population } \\
\hline & Speaker & Parents & \\
\hline 1 & \multirow{3}{*}{$\mathrm{D}_{\mathrm{F}}$} & Both $D_{F}$ & 60 \\
\hline 2 & & One $\mathrm{D}_{\mathrm{F}}$, one $\mathrm{D}_{\mathrm{IF}}$ & 55 \\
\hline 3 & & Both $D_{I F}$ & 19 \\
\hline 4 & \multirow{3}{*}{$\mathrm{D}_{\mathrm{IF}}$} & Both $D_{I F}$ & 66 \\
\hline 5 & & One $\mathrm{D}_{\mathrm{F}}$, one $\mathrm{D}_{\mathrm{IF}}$ & 21 \\
\hline 6 & & Both $D_{F}$ & 5 \\
\hline
\end{tabular}

Table 2 List of speakers' places of origin.

\begin{tabular}{ccc|ccc}
\hline Prefecture & Dialect & Population & Prefecture & Dialect & Population \\
\hline Aomori & $\mathrm{D}_{\mathrm{IF}}$ & 3 & Kyoto & $\mathrm{D}_{\mathrm{IF}}$ & 6 \\
Akita & $\mathrm{D}_{\mathrm{IF}}$ & 5 & Osaka & $\mathrm{D}_{\mathrm{IF}}$ & 16 \\
Miyagi & $\mathrm{D}_{\mathrm{F}}$ & 3 & Nara & $\mathrm{D}_{\mathrm{IF}}$ & 4 \\
Fukushima & $\mathrm{D}_{\mathrm{F}}$ & 3 & Wakayama & $\mathrm{D}_{\mathrm{IF}}$ & 1 \\
Ibaraki & $\mathrm{D}_{\mathrm{F}}$ & 3 & Hyogo & $\mathrm{D}_{\mathrm{IF}}$ & 10 \\
Tochigi & $\mathrm{D}_{\mathrm{F}}$ & 2 & Okayama & $\mathrm{D}_{\mathrm{IF}}$ & 3 \\
Gumma & $\mathrm{D}_{\mathrm{F}}$ & 4 & Hiroshima & $\mathrm{D}_{\mathrm{IF}}$ & 2 \\
Chiba & $\mathrm{D}_{\mathrm{F}}$ & 11 & Yamaguchi & $\mathrm{D}_{\mathrm{IF}}$ & 6 \\
Saitama & $\mathrm{D}_{\mathrm{F}}$ & 11 & Kagawa & $\mathrm{D}_{\mathrm{IF}}$ & 5 \\
Tokyo & $\mathrm{D}_{\mathrm{F}}$ & 40 & Tokushima & $\mathrm{D}_{\mathrm{IF}}$ & 2 \\
Kanagawa & $\mathrm{D}_{\mathrm{F}}$ & 25 & Ehime & $\mathrm{D}_{\mathrm{IF}}$ & 4 \\
Niigata & $\mathrm{D}_{\mathrm{F}}$ & 3 & Kochi & $\mathrm{D}_{\mathrm{IF}}$ & 3 \\
Toyama & $\mathrm{D}_{\mathrm{F}}$ & 3 & Fukuoka & $\mathrm{D}_{\mathrm{F}}$ & 9 \\
Ishikawa & $\mathrm{D}_{\mathrm{F}}$ & 2 & Nagasaki & $\mathrm{D}_{\mathrm{F}}$ & 3 \\
Aichi & $\mathrm{D}_{\mathrm{IF}}$ & 7 & Kumamoto & $\mathrm{D}_{\mathrm{F}}$ & 3 \\
Gifu & $\mathrm{D}_{\mathrm{IF}}$ & 5 & Oita & $\mathrm{D}_{\mathrm{F}}$ & 3 \\
Mie & $\mathrm{D}_{\mathrm{IF}}$ & 7 & Miyazaki & $\mathrm{D}_{\mathrm{F}}$ & 2 \\
Shiga & $\mathrm{D}_{\mathrm{IF}}$ & 3 & Kagoshima & $\mathrm{D}_{\mathrm{F}}$ & 4 \\
\hline
\end{tabular}

Target vowels were extracted from running speech by the linguistic units such as clauses and phrases. In the analysis, we excerpted as many target vowels as possible, making sure that we do not extract exactly the same clauses or phrases more than twice from a single speaker. As a result, the number of excerpted target vowels for one speaker ranged from 16 to 30 , with an average of 22.2 . Subsequently, we judged whether a target vowel was devoiced or not by inspecting waveforms and spectrograms. Figure 2 shows examples of the analysis. When there is no periodic cycle in the waveform and no glottal source pulse observed in the wide-band spectrogram, the target vowel was judged as "devoiced." When the vowel is partly devoiced or partly voiced, it was judged as "voiced." For all data, the judgment was conducted alone by the first author, who is a phonetically-trained native speaker of Japanese, in order to make the decisions consistent. We calculated the $P_{\mathrm{VD}}$ for each speaker by dividing the number 
(a)

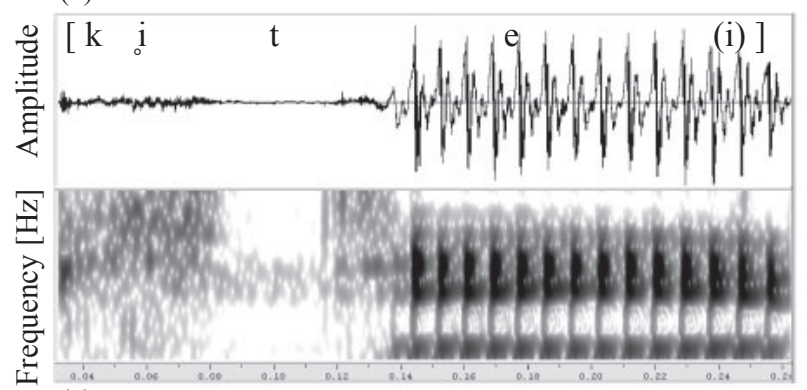

(c)

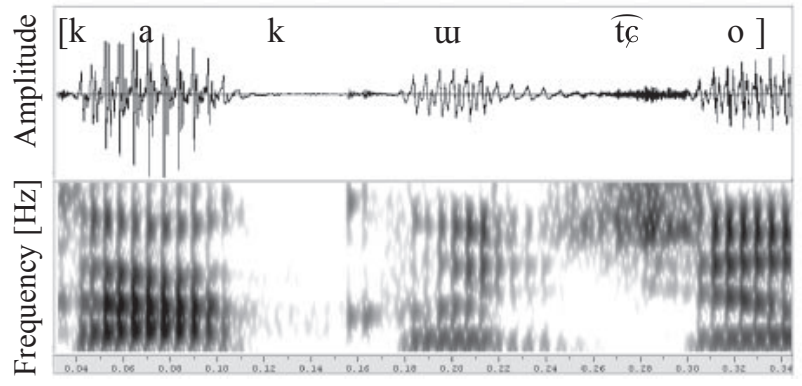

(b)

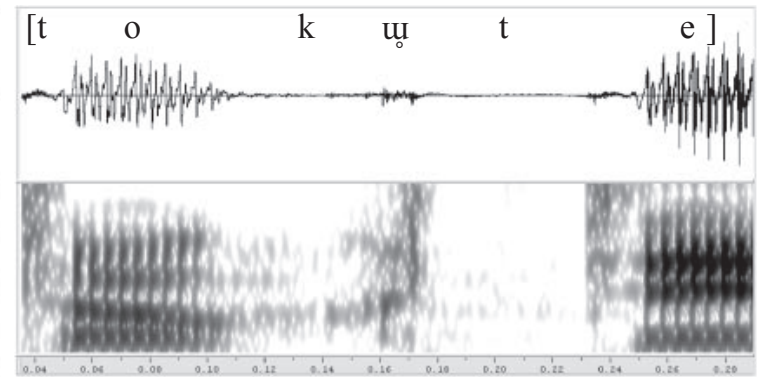

(d)

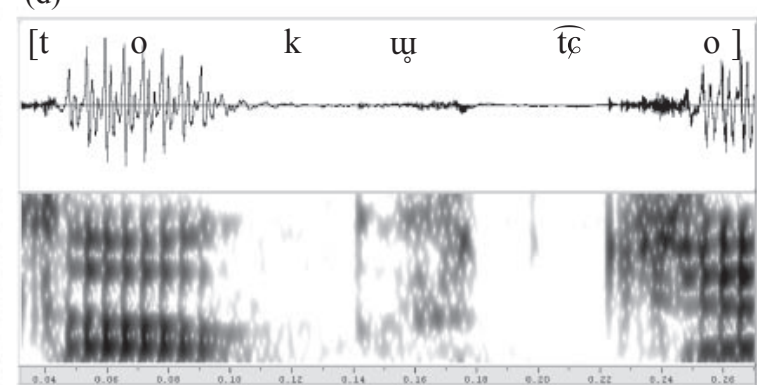

Time $[\mathrm{s}]$

Fig. 2 Examples of the analysis: (a) /kite(iru)/ (coming), (b) /tokute(:)/ (specific), (c) /kakut6o(:)/ (extension), (d) /tokut $\widehat{c}_{\mathrm{t}} \mathrm{O}(\mathrm{:}) /$ (characteristics). Only (c) was judged as 'voiced.'

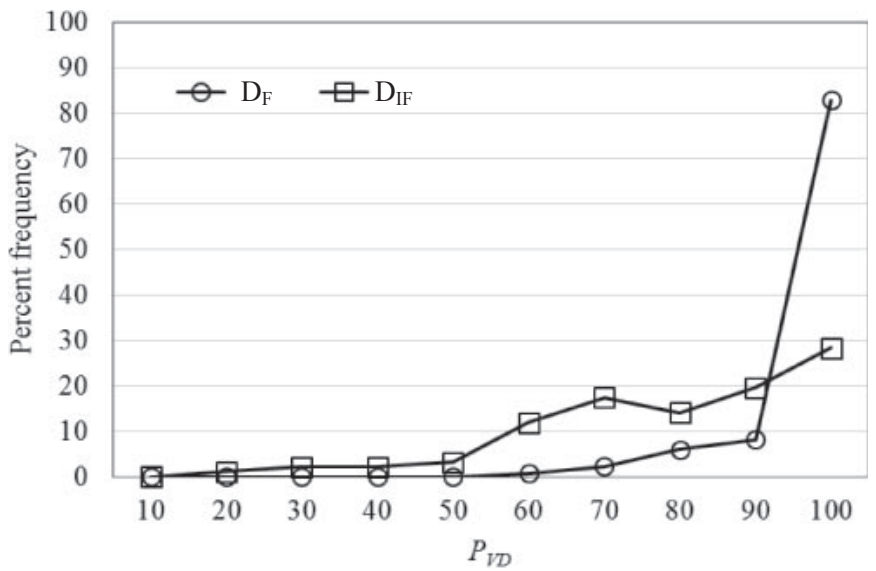

Fig. 3 Distribution of $P_{\mathrm{VD}}$ for each devoicing category. $\mathrm{D}_{\mathrm{F}}$ includes speakers of groups 1,2 , and 3 , and $\mathrm{D}_{\mathrm{IF}}$ includes speakers of groups 4,5 , and 6 .

of the devoiced vowels by that of the target vowels in order to use it as the measure for FVD.

\subsection{Results and Discussion}

3.2.1. Percentage of vowel devoicing for each speaker group

Figure 3 shows the distribution of $P_{\mathrm{VD}}$ values for the two devoicing categories, $\mathrm{D}_{\mathrm{F}}$ and $\mathrm{D}_{\mathrm{IF}}$. We can see that more than $80 \%$ of $\mathrm{D}_{\mathrm{F}}$ speakers (groups 1, 2, and 3) showed $100 \%$ devoicing, whereas the remaining $20 \%$ showed devoicing between $80 \%$ and $90 \%$. Speakers who showed devoicing less than $70 \%$ were rare. For $\mathrm{D}_{\mathrm{IF}}$ speakers (groups 4, 5, and 6), the distribution is widely spread
Table 3 Average percentage of vowel devoicing $\left(P_{\mathrm{VD}}\right)$ with standard deviation (S.D.) for each speaker group.

\begin{tabular}{cc}
\hline Speaker groups & Average percent devoiced (S.D.) \\
\hline 1 & $96.3(6.32)$ \\
2 & $92.7(9.50)$ \\
3 & $89.4(10.34)$ \\
4 & $70.7(19.02)$ \\
5 & $87.2(13.09)$ \\
6 & $84.8(17.46)$ \\
\hline
\end{tabular}

between $60 \%$ and $100 \%$. The effect of the parents' dialects was observed clearly. Table 3 shows the average $P_{\mathrm{VD}}$ and standard deviation of $P_{\mathrm{VD}}$ for each speaker group. $\mathrm{D}_{\mathrm{F}}$ speakers with $D_{F}$ parents devoiced the vowels the most, whereas $D_{\text {IF }}$ speakers with $D_{\text {IF }}$ parents devoiced them the least. Speakers with parents of unmatched devoicing categories showed in-between devoicing.

We examined whether we could estimate the dialect, or devoicing category, a speaker belongs to just by using $P_{\mathrm{VD}}$. Based on the data provided by Kawatsu and Maekawa [22] that investigated the average FVD of $\mathrm{D}_{\mathrm{F}}$ speakers of the same speech corpus as this study, the threshold between the two categories was set at $88.1 \%$.

The results of the category estimation are shown in Table 4. The average percentage of correct estimation for all speakers was $78.3 \%$ and that for the speakers of groups 1 and 4 was $87.3 \%$, whose devoicing category was matched with both of their parents'. The fact that the percentage for groups 1 and 4 is not $100 \%$ shows that some speakers show 
Table 4 Percentage of correct estimation of speakers' dialect (devoicing category) by using $P_{\mathrm{VD}}$.

\begin{tabular}{cc}
\hline Speaker group & Percent correct estimation \\
\hline 1 & 93.3 \\
2 & 81.8 \\
3 & 63.2 \\
4 & 81.8 \\
5 & 38.1 \\
6 & 40.0 \\
\hline
\end{tabular}

$P_{\mathrm{VD}}$ values that contradict their devoicing categories. It also means that we cannot predict a speaker's $P_{\mathrm{VD}}$ just from the speaker's and the parents' dialects. In order to find out the reasons why such speakers exist, we need to investigate the actual $P_{\mathrm{VD}}$ of the speakers' parents and probably even that of their grandparents. In any generation, there may be a proportion of speakers whose $P_{\mathrm{VD}}$ contradicts the devoicing category of the dialect. Furthermore, we have to consider the effects of other factors on speakers' $P_{\mathrm{VD}}$, such as mass media, local community, and people we meet at school. Further investigation of these factors from various perspectives is needed.

On comparing the percentage of correct category estimation, speakers of $\mathrm{D}_{\mathrm{F}}$ obtained a better score than those of $\mathrm{D}_{\mathrm{IF}}$. There was a difference between these two categories in the history of moving. Only $14.9 \%$ of $\mathrm{D}_{\mathrm{F}}$ speakers had experienced moving to the $\mathrm{D}_{\mathrm{IF}}$ districts, whereas $51.1 \%$ of $\mathrm{D}_{\mathrm{IF}}$ speakers had moved to $\mathrm{D}_{\mathrm{F}}$ districts.
When we exclude those who had moved to the district of different devoicing category, the percentage of correct category estimation for $\mathrm{D}_{\mathrm{F}}$ speakers was $85.5 \%$ (improved from $84.3 \%$ ) and that for $\mathrm{D}_{\mathrm{IF}}$ speakers was $80.0 \%$ (improved from 69.6\%). As for the influence of the history of moving on $P_{\mathrm{VD}}$, we need further investigation in future, since we did not have speakers with history of moving during the critical period of language acquisition.

3.2.2. Effect of linguistic and social factors

Average $P_{\mathrm{VD}}$ was calculated for $\mathrm{D}_{\mathrm{F}}$ and $\mathrm{D}_{\mathrm{IF}}$ speakers and for different $\mathrm{C}_{1}$ and $\mathrm{C}_{2}$ combinations, as shown in Table 5. From the table, the overall average $P_{\mathrm{VD}}$ for $\mathrm{D}_{\mathrm{F}}$ was $97.0 \%$ for the front vowel /i/ and $89.9 \%$ for the back vowel / $\mathrm{w} /$, whereas that for $\mathrm{D}_{\mathrm{IF}}$ was $63.8 \%$ for $/ \mathrm{i} /$, excluding the affricate-/i/-affricate condition that had only one token, and $61.8 \%$ for $/ \mathrm{w} /$. In both the dialects, the front vowel was slightly more often devoiced than the back vowel. For the speakers of $\mathrm{D}_{\mathrm{F}}, P_{\mathrm{VD}}$ was high when $\mathrm{C}_{2}$ was a plosive. This tendency was also confirmed by other studies for both words [2,11,12] and running speech [22]. For speakers of $\mathrm{D}_{\mathrm{IF}}$, as well, the same tendency was observed, which corroborates the report by Kawatsu and Maekawa [22].

Generally speaking, the faster the speech, the more the vowel devoicing [2]. We analysed the average articulation rate (morae per second) of the excerpted utterances for each speaker and examined the relationship between speech rate and vowel devoicing. Correlation coefficients were calculated between average articulation rate and the

Table 5 Average $P_{\mathrm{VD}}$ for each consonantal context for (a) speakers of $\mathrm{D}_{\mathrm{F}}$ and (b) speakers of $\mathrm{D}_{\mathrm{IF}}$. $\mathrm{C}_{1}$ and $\mathrm{C}_{2}$ stand for preceding and following consonants, respectively. Affr., Fric., and Plos., and Ave. stand for affricates, fricatives, plosives, and average, respectively.

(a)

\begin{tabular}{ccccccc}
\hline \multirow{2}{*}{$(n=964)$} & \multicolumn{3}{c}{$\mathrm{C}_{2}$} & \multirow{2}{*}{ Ave. } \\
\cline { 3 - 6 } & Affr. & Affr. & Fric. & Plos. & \\
\hline \multirow{3}{*}{$\mathrm{C}_{1}$} & Fric. & $100^{1)}$ & - & 100 & 100 \\
\cline { 2 - 6 } & & Plos. & 100 & - & 98.9 & 99.4 \\
\hline & Ave. & 94.7 & 91.0 & 94.7 & 93.5 \\
\hline
\end{tabular}

(b)

\begin{tabular}{|c|c|c|c|c|c|}
\hline \multirow{2}{*}{\multicolumn{2}{|c|}{$\begin{array}{c}/ \mathrm{i} / \\
(n=815)\end{array}$}} & \multicolumn{3}{|c|}{$\mathrm{C}_{2}$} & \multirow{2}{*}{ Ave. } \\
\hline & & Affr. & Fric. & Plos. & \\
\hline \multirow{3}{*}{$\mathrm{C}_{1}$} & Affr. & $0.0^{1,2)}$ & - & 81.8 & 81.8 \\
\hline & Fric. & 69.6 & - & 91.4 & 80.5 \\
\hline & Plos. & 40.0 & 36.1 & 63.9 & 46.7 \\
\hline \multicolumn{2}{|c|}{ Ave. } & 54.8 & 36.1 & 79.1 & 63.8 \\
\hline
\end{tabular}

1) Affricate-vowel-affricate condition had only a few tokens in both dialects: eight tokens for (a)-/i/, five for (a)-/w/, one for (b)-/i/, and five for (b)-/u/.

2) This is not included in the calculation of average, as only one token was available for the analysis.

\begin{tabular}{cccccc}
\hline \multirow{2}{*}{$\begin{array}{c}\text { (n) } \\
(n=905)\end{array}$} & \multicolumn{3}{c}{$\mathrm{C}_{2}$} & \multirow{2}{*}{ Ave. } \\
\cline { 3 - 5 } & Affr. & Fric. & Plos. & \\
\hline \multirow{3}{*}{$\mathrm{C}_{1}$} & Affr. & $60.0^{1)}$ & - & 98.4 & 79.2 \\
\cline { 2 - 5 } & Fric. & 98.0 & - & 97.2 & 97.6 \\
\cline { 2 - 5 } & Plos. & 88.5 & 90.8 & 96.0 & 91.8 \\
\hline \multicolumn{2}{c}{ Ave. } & 82.2 & 90.8 & 97.2 & 89.9 \\
\hline
\end{tabular}

\begin{tabular}{cccccc}
\hline \multirow{2}{*}{$(n=878)$} & \multicolumn{4}{c}{$\mathrm{C}_{2}$} & \multirow{2}{*}{ Ave. } \\
\cline { 3 - 5 } & Affr. & Fric. & Plos. & \\
\hline \multirow{3}{*}{$\mathrm{C}_{1}$} & Affr. & $66.7^{1)}$ & - & 84.2 & 75.5 \\
\cline { 2 - 5 } & Fric. & 55.8 & - & 72.9 & 64.3 \\
\cline { 2 - 5 } & Plos. & 47.7 & 56.4 & 48.7 & 50.9 \\
\hline \multicolumn{2}{c}{ Ave. } & 56.7 & 56.4 & 68.6 & 61.8 \\
\hline
\end{tabular}


speaker's $P_{\mathrm{VD}}$. No significant tendency was observed for speakers of $\mathrm{D}_{\mathrm{F}}(r=0.11, d . f .=132, p=0.19)$. On the other hand, the data for $\mathrm{D}_{\mathrm{IF}}$ speakers showed a significant positive correlation, although it was weak $(r=0.23$, $d . f .=90, p=0.03)$. This does not corroborate Fujimoto's claim that although both dialect and speech rate affect percentage of devoicing, they do not have interaction with each other [2]. However, it still supports a second claim by her [12] that vowel devoicing is a phonetic, not phonological, process for $\mathrm{D}_{\mathrm{IF}}$ speakers.

Using two-way analysis of variance (ANOVAs), the effects of generation and gender on $P_{\mathrm{VD}}$ were investigated. To investigate the effect of generation, we divided the speakers into four generations according to their year of birth, i.e. before 1964, between 1965 and 1969, between 1970 and 1974, and after 1975. The results showed that neither generation $(F(3,3)=5.96, p=0.09)$ nor gender $(F(1,3)=1.89, p=0.26)$ had a significant effect on $P_{\mathrm{VD}}$. In Imai's study [15], younger male speakers devoiced the vowels more than younger female speakers did. This tendency was not observed in this study with all of the speakers; however, when we analyse only $\mathrm{D}_{\mathrm{F}}$ speakers as in Imai's study, the same tendency was found $(F(1,3)=11.7$, $p=0.04)$. The effect of generation was yet not significant $(F(3,3)=1.92, p=0.30)$; this may be due to the difference in the ways of grouping the speakers.

\section{WITHIN-SPEAKER REPRODUCIBILITY}

\subsection{Analysis Methods}

A subset of the speech materials analysed in Sect. 3 was used for analysing within-speaker reproducibility. A total of 50 speakers, 25 whose $P_{\mathrm{VD}}$ was above the threshold (hereafter, frequent speakers) and 25 whose $P_{\mathrm{VD}}$ was below it (hereafter, infrequent speakers), were selected at random. Based on their dialects, there were $22 \mathrm{D}_{\mathrm{F}}$ and $28 \mathrm{D}_{\mathrm{IF}}$ speakers. In order to investigate the relationship between $P_{\mathrm{VD}}$, dialects, and the reproducibility of vowel devoicing, we focused on the segmental contexts where significant dialectal differences would appear, which means forensically more prospective, i.e. the context where $C_{1}$ is a plosive. In this context, the average $P_{\mathrm{VD}}$ values for $\mathrm{D}_{\mathrm{F}}$ and $\mathrm{D}_{\mathrm{IF}}$ were $96.4 \%(n=307)$ and $47.4 \%(n=388)$, respectively. Among them, we concentrated on the segmental contexts that would occur frequently, i.e. where $\mathrm{C}_{1}$ is the velar plosive $/ \mathrm{k} /$ and $\mathrm{C}_{2}$ is $/ \mathrm{t} /, / \mathrm{k} /$, or $/ \mathrm{s} /$.

We used the text browser MonoForC, which was attached to CSJ, and scanned the target segmental contexts for all speech samples. Subsequently, we selected the words or phrases that were uttered by the same speaker more than twice and analysed the FVD. We applied the same analysis method as in Sect. 3.1. The average number of analysed samples in types (words and phrases) was 9.2 for one speaker (ranged from 1 to 28 , median 8.5, mode 4) and that of repetitions in tokens (utterances) was 4.4 (ranged from 2 to 49, median 3, mode 2).

In order to evaluate the reproducibility of vowel devoicing, we used a measure $R$, which is defined as follows:

$$
R=\frac{\left|n_{\mathrm{D}}-n_{\mathrm{V}}\right|}{n_{\mathrm{U}}},
$$

where $n_{\mathrm{U}}$ is the number of utterances for one target word or phrase, $n_{\mathrm{D}}$ is the number of samples where vowel devoicing occurred, and $n_{\mathrm{V}}$ is the number of samples where vowel devoicing did not occur. The closer $R$ is to 1 , the higher is the reproducibility.

\subsection{Results and Discussion}

\subsubsection{Relationship between $P_{\mathrm{VD}}$ and reproducibility}

The average values of $P_{\mathrm{VD}}$ and $R$ for all the analysed speech samples were $89.1 \%$ and $0.93(n=205)$, respectively, for frequent speakers, and $68.4 \%$ and $0.80 \quad(n=$ 254), respectively, for infrequent speakers. We can see that frequent speakers consistently devoiced the vowels in most of the target contexts, whereas infrequent speakers devoiced some of the vowels only relatively occasionally. The results of the Welch's test revealed that the differences between the two speaker groups were significant for both $P_{\mathrm{VD}}(t(453.5)=3.48, p<0.001)$ and $R(t(429.7)=7.07$, $p<0.001)$.

For frequent speakers, $P_{\mathrm{VD}}$ was appreciably lower when the following mora was $/ \mathrm{si} /(M=59.5, n=37)$ compared to other segmental contexts $(M=88.4, n=$ 129). For infrequent speakers, as well, a similar tendency was observed $(M=44.7, n=38$ before $/ \mathrm{si} /$, and $M=$ $59.0, n=166$ for other contexts), although the difference was smaller. We also found that for both speaker groups, devoicing occurred inconsistently when pause was inserted for indicating a focus or before a citation, although such speech samples were omitted from the analysis in this study.

\subsubsection{Effect of the dialects}

When we classify speakers according to their devoicing categories, the between-group differences in the average $P_{\mathrm{VD}}$ and $R$ values were smaller than when we classify the speakers according to their $P_{\mathrm{VD}}$ : the $P_{\mathrm{VD}}$ and $R$ values for $\mathrm{D}_{\mathrm{F}}$ speakers were $85.0 \%$ and 0.89 , respectively, whereas those for $\mathrm{D}_{\mathrm{IF}}$ speakers were $69.4 \%$ and 0.84 , respectively. The results of the Welch's test showed that the difference between the two dialects was significant only for $P_{\mathrm{VD}}$ $(t(359.7)=4.49, p<0.001)$ and not for $R(t(384.2)=$ $1.82, p=0.07)$.

So far, vowel devoicing has been investigated only for native speakers and been discussed as being a phonological process for one dialect and a phonetic process for another [e.g. 12]; however, our results imply that we had better 
discuss it from the perspective of $P_{\mathrm{VD}}$. Namely, whereas vowel devoicing may be a phonological process for frequent speakers, it is a phonetic process for infrequent speakers.

\section{SUMMARY AND CONCLUDING REMARKS}

In this study, we analysed the spontaneous speech uttered by 226 speakers in order to investigate how the places of origin of the speakers and their parents influence FVD and to provide reference data on FVD of Japanese spontaneous speech. Three major findings were obtained. Firstly, whereas $D_{F}$ speakers whose parents also speak $D_{F}$ devoiced the vowels the most, $\mathrm{D}_{\mathrm{IF}}$ speakers whose parents also speak $\mathrm{D}_{\mathrm{IF}}$ devoiced them the least. Speakers with parents of unmatched devoicing categories showed devoicing that was between that of genuine $\mathrm{D}_{\mathrm{F}}$ and $\mathrm{D}_{\mathrm{IF}}$ speakers.

Secondly, when we estimate the speakers' devoicing categories by using $P_{\mathrm{VD}}$, the percentage of correct estimation for speakers with the parents of matched category was $87.3 \%$. This means that some speakers showed $P_{\mathrm{VD}}$ values that contradicts their devoicing category. The existence of such speakers has not been reported before; however, it is important in forensics. We should not be too ready to judge whether a speaker comes from the district of $\mathrm{D}_{\mathrm{F}}$ or $\mathrm{D}_{\mathrm{IF}}$ just by looking at the speaker's $P_{\mathrm{VD}}$. Instead, we should also carefully examine other dialect-related features, such as the phonemic system or the accent patterns that the speaker uses. In our study, the proportion of speakers with contradicting $P_{\mathrm{VD}}$ was larger among $\mathrm{D}_{\mathrm{IF}}$ speakers. Regarding the history of moving, more $\mathrm{D}_{\mathrm{IF}}$ compared to $\mathrm{D}_{\mathrm{F}}$ speakers had an experience of moving to the other devoicing category district. Acquisition of vowel devoicing is said to finish before a speaker reaches 5 years old [16]; however, no research has yet been done on the acquisition of vowel devoicing after the critical period. Furthermore, we found that some $\mathrm{D}_{\mathrm{IF}}$ speakers showed almost $100 \% P_{\mathrm{VD}}$ and still kept the accentuation and intonation of their own dialects. These speakers must be further analysed to know about the acquisition and the development of mother dialects. As a future direction of this study, we will analyse speech samples of the speakers who experienced moving to different dialect districts during the language critical period. We will also investigate the effect of the accentuation system and the relationship between the speaker's social and linguistic backgrounds and $P_{\mathrm{VD}}$.

Finally, in the second half of this study, we examined the within-speaker variability of vowel devoicing. We analysed the speech samples of 50 speakers, focusing on the segmental context where significant dialectal difference was observed in Sect. 3, i.e. the target vowel positioned between $/ \mathrm{k} /$ as $\mathrm{C}_{1}$ and $/ \mathrm{t} /, / \mathrm{k} /$, or $/ \mathrm{s} /$ as $\mathrm{C}_{2}$. We found that frequent speakers, whose $P_{\mathrm{VD}}$ was above the threshold, devoiced most of the target vowels consistently, whereas infrequent speakers, whose $P_{\mathrm{VD}}$ was below the threshold, devoiced them only occasionally and inconsistently. When re-arranging the speaker groups according to their dialects, although the difference in $P_{\mathrm{VD}}$ was kept significant, that in $R$ was not. This suggests that we should discuss the phenomenon of vowel devoicing in terms of $P_{\mathrm{VD}}$; whereas is a phonological process for frequent speakers, it is a phonetic process for infrequent speakers. Forensically, when we obtain two speech samples and both of them show $P_{\mathrm{VD}}$ that is high enough, we should also analyse the reproducibility. If one of them clearly shows consistently frequent vowel devoicing, while the other shows inconsistent devoicing, then we can say that there is a possibility that the two speech samples were produced by two different speakers. Further investigation is necessary regarding whether speakers can disguise their speech by making a consistent change in vowel devoicing. Except for phoneticians, most people may not be aware of whether they are devoicing the vowels or not by themselves. We should also examine the effects of sociologically different circumstances on vowel devoicing. In many languages, within-speaker variation in pronunciation is reported to be a function of the degree of formality [24,25]. This study involves relatively formal speech, which is almost as formal as reading, and we should further investigate how it differs from speech that is more casual.

\section{ACKNOWLEDGMENTS}

This study summarises a series of work conducted by the authors that were presented at the annual meetings of the Acoustical Society of Japan [26-28]. This work was supported by Grants-in-Aid for Scientific Research by MEXT (26870865, 26350466, 25350488, 25280066, 24710195).

\section{REFERENCES}

[1] H. G. Byun, "Regional and generational differences of high vowel devoicing in Japanese," Stud. Jpn. Lang., 3(1), pp. 3348 (2007) (in Japanese).

[2] M. Fujimoto and S. Kiritani, "Comparison of vowel devoicing for speakers of Tokyo- and Kinki dialects," J. Phon. Soc. Jpn., 7(1), pp. 58-69 (2003) (in Japanese).

[3] M. Sugito, The Sounds of Japanese (Izumishoin, Osaka, 1996) (in Japanese).

[4] NHK Broadcasting Culture Research Institute, The Japanese Language Pronunciation and Accent Dictionary (NHK Publishing, Tokyo, 1998) (in Japanese).

[5] I. Konishi, "Research approach for dialects in new age," in Research Methods in Dialectology, T. Kobayashi, Ed. (Iwanamishoten, Tokyo, 2007), pp. 1-38 (in Japanese).

[6] M. Jessen, "Forensic reference data on articulation rate in German," Sci. Justice, 47, 50-67 (2007).

[7] Y. Kinoshita and S. Ishihara, "Background population: How does it affect LR-based forensic voice comparison?," Int. J. Speech Lang. Law, 21, 191-224 (2014). 
[8] P. Rose, Forensic Speaker Identification (Taylor and Francis, New York, 2002).

[9] T. Vance, The Sounds of Japanese (Cambridge University Press, Cambridge, 2008).

[10] H. Kawai, N. Higuchi, T. Shimizu and S. Yamamoto, "Devoicing rules for text-to-speech synthesis of Japanese," J. Acoust. Soc. Jpn. (J), 51, 698-705 (1995) (in Japanese).

[11] N. Yoshida, "The effects of phonetic environment on vowel devoicing in Japanese," Kokugogaku, 53(3), pp. 34-47 (2002) (in Japanese).

[12] M. Fujimoto, "Vowel duration and vowel devoicing in Japanese: A comparison between Tokyo and Osaka dialect speakers," Kokugogaku, 55(1), pp. 2-15 (2004) (in Japanese).

[13] M. Fujimoto, "Vowel devoicing," in Handbook of Japanese Phonetics and Phonology, H. Kubozono, Ed. (De Gruyter, Berlin, 2015), Chap. 4, pp. 167-214.

[14] H. G. Byun, "An age analysis of devoicing rules in five Japanese dialects," Stud. Jpn. Lang., 6(4), pp. 79-94 (2010) (in Japanese).

[15] T. Imai, "An emerging gender difference in Japanese vowel devoicing," in A Reader in Sociophonetics, D. R. Preston and N. Niedzielski, Eds. (De Gruyter, New York, 2010), Chap. 6, pp. 177-190.

[16] S. Imaizumi, K. Fuwa and H. Hosoi, "Development of adaptive phonetic gestures in children: Evidence from vowel devoicing in two different dialects of Japanese," J. Acoutst. Soc. Am., 106, 1033-1044 (1999).

[17] M. Komatsu and M. Aoyagi, "Vowel devoicing vs. mora-timed rhythm in spontaneous Japanese: Inspection of phonetic labels of OGI_TS,” Proc. Interspeech 2005, pp. 2461-2464 (2005).

[18] K. Maekawa and H. Kikuchi, "Corpus-based analysis of vowel devoicing in spontaneous Japanese: An interim report," in Voicing in Japanese, J. Van de Weijer, K. Nanjo and T. Nishihara, Eds. (De Gruyter, New York, 2005), pp. 205-228.

[19] N. Tsujimura, An Introduction to Japanese Linguistics (Blackwell, Oxford, 1996).

[20] M. Fujimoto, "Glottal opening patterns in devoiced tokens by an Osaka dialect speaker," J. Phon. Soc. Jpn., 9(1), pp. 50-59 (2005) (in Japanese).

[21] K. Okamoto, "Vowel devoicing of Osaka dialect speakers: On its individual characteristics," Machikaneyama Ronso Japanology, 39, 37-53 (2005) (in Japanese).

[22] H. Kawatsu and K. Maekawa, "Influence of the manner of articulation on vowel devoicing rate: Analysis of the Corpus of Spontaneous Japanese," Proc. Spring Meet. Acoust. Soc. Jpn., pp. 443-444 (2009) (in Japanese).

[23] K. Maekawa, "An overview of CSJ," http://www.ninjal.ac.jp/ corpus_center/csj/manu-f/overview.pdf (accessed 2016-03-08) (in Japanese).

[24] W. Labov, "Sources of inherent variation in the speech process," in Invariance and Variability in Speech Processes, J. S. Perkell and D. H. Klatt, Eds. (Psychology Press, Oxford,
1986), pp. 401-425.

[25] V. G. Walker, "Durational characteristics of young adults during speaking and reading tasks," Folia Phoniatr., 40, 12-20 (1988).

[26] K. Amino, H. Makinae, T. Kamada and T. Osanai, "Relationship between occurrence frequency of vowel devoicing and speaker's place of origin," Proc. Spring Meet. Acoust. Soc. Jpn., pp. 381-384 (2013) (in Japanese).

[27] K. Amino, H. Makinae, T. Kamada and T. Osanai, "Effect of the speaker's and parents' birthplace on the occurrence frequency of vowel devoicing," Proc. Autumn Meet. Acoust. Soc. Jpn., pp. 271-274 (2013) (in Japanese).

[28] K. Amino, H. Makinae, T. Kamada and T. Osanai, "Withinspeaker reproducibility of vowel devoicing," Proc. Spring Meet. Acoust. Soc. Jpn., pp. 507-510 (2014) (in Japanese).

Kanae Amino received the B.A. and M.A. degrees in linguistics and Ph.D. degree in engineering from Sophia University, Tokyo, Japan, in 2002, 2004, and 2009, respectively. She was a post-doctoral researcher at Sophia University in 2009-2010 and a JSPS Research Fellow (PD) in 2010-2012. Currently she is a researcher at National Research Institute of Police Science (NRIPS) in Chiba, Japan. She has been working on the variations in speech production and the perception of the speaker individualities. Her research interests also include Japanese phonology and phonetics, and dialect and language identification for forensic purposes.

Hisanori Makinae received the B.E., M.E. and Ph.D. degrees in Electrical and Communication Engineering from Tohoku University, Miyagi, Japan, in 1998, 2000 and 2008, respectively. Since 2005, he has been with NRIPS, Chiba, Japan, where he is currently a senior researcher of Third Information Science Section. His research interests include speaker recognition in forensic field. He is a member of ASJ and the Japanese Association of Forensic Science and Technology (JAFST).

Toshiaki Kamada received the B.E. degree in electrical engineering from the University of Tokyo, Tokyo, Japan, in 1995. He has been with NRIPS, Chiba, Japan, since 1995. His research interests include speaker recognition and speech processing in forensic field. $\mathrm{He}$ is a member of ASJ and JAFST.

Takashi Osanai received the B.E., M.E. and Ph.D. degrees in electro-communications from University of Electro-Communications, Tokyo, Japan, in 1985, 1987 and 2007, respectively. Since 1987, he has been with NRIPS, Chiba, Japan, where he is currently a director of Identification Centre. In 2001, he has been a visiting fellow at the Phonetics Laboratory in the Department of Linguistics at the Australian National University, Canberra, Australia. His current research interests include speaker recognition in forensic field. 